\title{
Combined hepatocellular-cholangiocarcinoma: which preoperative clinical data and conventional MRI characteristics have value for the prediction of microvascular invasion and clinical significance?
}

\author{
Xiaolong Wang ${ }^{1,2,3} \cdot$ Wentao Wang ${ }^{1} \cdot$ Xijuan $\mathrm{Ma}^{4} \cdot \mathrm{Xin}_{\mathrm{Lu}}{ }^{2,3} \cdot$ Shaodong $\mathrm{Li}^{2,3} \cdot$ Mengsu Zeng ${ }^{1} \cdot \mathrm{Kai}^{\mathrm{X}} \mathrm{Uu}^{2,3} \cdot$ Chun Yang $^{1}$
}

Received: 14 February 2020 / Revised: 13 March 2020 / Accepted: 2 April 2020 / Published online: 8 May 2020

(C) The Author(s) 2020

\begin{abstract}
Objectives To explore which preoperative clinical data and conventional MRI findings may indicate microvascular invasion (MVI) of combined hepatocellular-cholangiocarcinoma (cHCC-CCA) and have clinical significance.

Methods The study enrolled 113 patients with histopathologically confirmed cHCC-CCA (MVI-positive group [ $n=56]$, MVInegative group $[n=57]$ ). Two radiologists retrospectively assessed the preoperative MRI features (qualitative analysis of morphology and dynamic enhancement features), and each lesion was assigned according to the LI-RADS. Preoperative clinical data were also evaluated. Logistic regression analyses were used to assess the relative value of these parameters as potential predictors of MVI. Recurrence-free survival (RFS) rates after hepatectomy in the two groups were estimated using Kaplan-Meier survival curves and compared using the log-rank test.

Results The majority of cHCC-CCAs were categorized as LR-M. On multivariate analysis, a higher serum AFP level (OR, $0.523 ; 95 \% \mathrm{CI}, 0.282-0.971 ; p=0.040)$, intratumoral fat deposition (OR, 14.368; 95\% CI, 2.749-75.098; $p=0.002)$, and irregular arterial peritumoral enhancement (OR, $0.322 ; 95 \% \mathrm{CI}, 0.164-0.631 ; p=0.001)$ were independent variables associated with the MVI of cHCC-CCA. After hepatectomy, patients with MVI of cHCC-CCA showed earlier recurrence than those without MVI (hazard ratio [HR], 0.402; 95\% CI, 0.189-0.854, $p=0.013$ ).

Conclusion A higher serum AFP level and irregular arterial peritumoral enhancement are potential predictive biomarkers for the MVI of cHCC-CCA, while intratumoral fat detected on MRI suggests a low risk of MVI. Furthermore, cHCC-CCAs with MVI may have worse surgical outcomes with regard to early recurrence than those without MVI.

Key Points

- Higher serum levels of AFP combined with irregular arterial peritumoral enhancement are independent risk factors for the MVI of cHCC-CCA, while fat deposition might be a protective factor.

- $\mathrm{HCC}$-CCA with MVI may have a higher risk of early recurrence after surgery.

- Most cHCC-CCAs were categorized as LR-M in this study, and no significant difference was found in MVI based on LI-RADS category.
\end{abstract}

Keywords Liver neoplasms $\cdot$ Neoplasm invasiveness $\cdot$ Magnetic resonance imaging

Xiaolong Wang and Wentao Wang contributed equally to this work.

Kai Xu

xkpaper@163.com

Chun Yang

dryangchun@hotmail.com

1 Department of Radiology, Zhongshan Hospital, Fudan University, No. 180 Fenglin Road, Xuhui District, Shanghai, China
2 Department of Radiology, The Affiliated Hospital of Xuzhou Medical University, No. 99 Huaihai West Road, Xuzhou, Jiangsu Province, China

3 School of Medical Imaging, Xuzhou Medical University, Xuzhou, Jiangsu Province, China

4 Department of Radiology, Xuzhou Central Hospital, Xuzhou, Jiangsu Province, China 


$\begin{array}{ll}\text { Abbreviations } & \\ \text { AFP } & \text { Alpha-fetoprotein } \\ \text { APHE } & \text { Arterial phase hyperenhancement } \\ \text { CA19-9 } & \text { Cancer antigen 19-9 } \\ \text { CEA } & \text { Carcinoembryonic antigen } \\ \text { cHCC-CCA } & \begin{array}{l}\text { Combined hepatocellular- } \\ \text { cholangiocarcinoma }\end{array} \\ \text { CI } & \text { Confidence interval } \\ \text { DWI } & \text { Diffusion-weighted imaging } \\ \text { Gd-DTPA } & \text { Gadopentate dimeglumine } \\ \text { HBP } & \text { Hepatobiliary phase } \\ \text { HCC } & \text { Hepatocellular carcinoma } \\ \text { ICC } & \text { Intrahepatic cholangiocarcinoma } \\ \text { LI-RADS } & \text { Liver Imaging Reporting and Data System } \\ \text { LR-M } & \text { Probably or definitely malignant, } \\ & \text { not HCC specific } \\ \text { MRI } & \text { Magnetic resonance imaging } \\ \text { MVI } & \text { Microvascular invasion } \\ \text { NPV } & \text { Negative predictive value } \\ \text { OR } & \text { Odds ratio } \\ \text { OS } & \text { Overall survival } \\ \text { PLCs } & \text { Primary liver carcinomas } \\ \text { PPV } & \text { Positive predictive value } \\ \text { RFS } & \text { Recurrence-free survival } \\ \text { TACE } & \text { Transarterial chemoembolization }\end{array}$

\section{Introduction}

Combined hepatocellular-cholangiocarcinoma (cHCC-CCA) is a relatively uncommon subtype of primary hepatic malignant tumors, accounting for 2-5\% of primary liver carcinomas (PLCs) [1-3]. Some studies have shown that cHCC-CCA has a biological behavior and prognosis that are intermediate between those of hepatocellular carcinoma (HCC) and intrahepatic cholangiocarcinoma (ICC) [4]; however, some reports have also stated that $\mathrm{cHCC}-\mathrm{CCA}$ has a significantly worse prognosis than $\mathrm{HCC}$ and ICC, even after curative resection [5]. At present, the risk factors identified as being related to prognosis of cHCC-CCA are not uniform across studies because of the relatively low incidence and variations in sample size. Currently, studies have indicated that vascular invasion, lymph node metastasis, satellite nodules, and tumor size are major predictive factors for the prognosis of $\mathrm{cHCC}$ CCA [6-8]. Studies have also shown that the level of cancer antigen 19-9 (CA19-9) or the presence of cirrhosis is a factor affecting the prognosis of cHCC-CCA $[9,10]$. Scholars have not yet come to a consensus regarding the prognostic factors of cHCC-CCA. Although previous studies have confirmed that microvascular invasion (MVI) is a prognostic factor for tumor recurrence and is associated with poor survival outcomes in HCC [11-14] and ICC [15, 16], the relationship between prognosis and the presence of MVI in cHCC-CCA patients has not yet been established.

Currently, multiple magnetic resonance imaging (MRI) techniques have been used to improve the preoperative prediction of MVI in HCC [17-21]. Some imaging findings, such as "arterial peritumoral enhancement," "tumor margin," and "peritumoral hypointensity on hepatobiliary phase (HBP)," have been reported to be related to MVI in HCC [20]; some studies have shown that "incomplete tumor capsule" has a significant relationship with MVI in HCC [21]. A small number of studies have also used MRI to predict the MVI of massforming intrahepatic cholangiocarcinoma [22]. Currently, almost all the existing MRI studies have only described the imaging features or clinical characteristics of cHCC-CCA compared to those of pure HCC and ICC, usually with a small sample size [23-29]. Recently, studies have utilized LR-M features (including rim arterial phase hyperenhancement (APHE), peripheral "washout" appearance and delayed central enhancement) defined in version 2017 of the Liver Imaging Reporting and Data System (LI-RADS) to identify cHCC-CCA and HCC, and shown that LI-RADS categorization may provide prognostic information on cHCC-CCAs after surgery $[30,31]$. However, these studies did not attempt to identify valuable preoperative MRI features indicating MVI in cHCC-CCA patients. Therefore, the purpose of this study was to evaluate the value of preoperative clinical data and conventional MRI findings including morphology, enhanced features, and the LI-RADS category for the preoperative prediction of the MVI of cHCC-CCA. Furthermore, the effect of MVI risk on the early recurrence of cHCC-CCA after surgery was estimated by the follow-up recurrence-free survival (RFS).

\section{Materials and methods}

\section{Patient selection}

This retrospective study was approved by our institutional review board, and the need for informed patient consent was waived. Between January 2016 and June 2019, in total, 192 consecutive patients were confirmed by postoperative pathology to have cHCC-CCA and without extrahepatic metastasis by preoperative examinations. The inclusion criteria were as follows: (a) primary liver lesions without any prior treatment; (b) the MRI examinations were performed within 30 days before hepatectomy, and the MRI scans satisfied the diagnostic criteria; (c) there was a single mass without intrahepatic metastasis or lesions with multiple origins; and (d) the maximum diameter of the lesion was $\geq 1 \mathrm{~cm}$. Finally, 79 cases were excluded for the following reasons: previous treatment history ( $n=17,8$ cases of hepatectomy and 9 cases of transarterial chemoembolization [TACE] therapy); no MRI scans within 
Fig. 1 Flowchart detailing the patient selection process and exclusion criteria. In total, 113 patients with cHCC-CCA were enrolled in the final analysis. cHCC-CCA, combined hepatocellular-

cholangiocarcinoma; MRI, magnetic resonance imaging; MVI, microvascular invasion
Consecutive patients with pathologically confirmed cHCC-CCA

after hepatectomy between January 2016 to June 2019 (n=192)

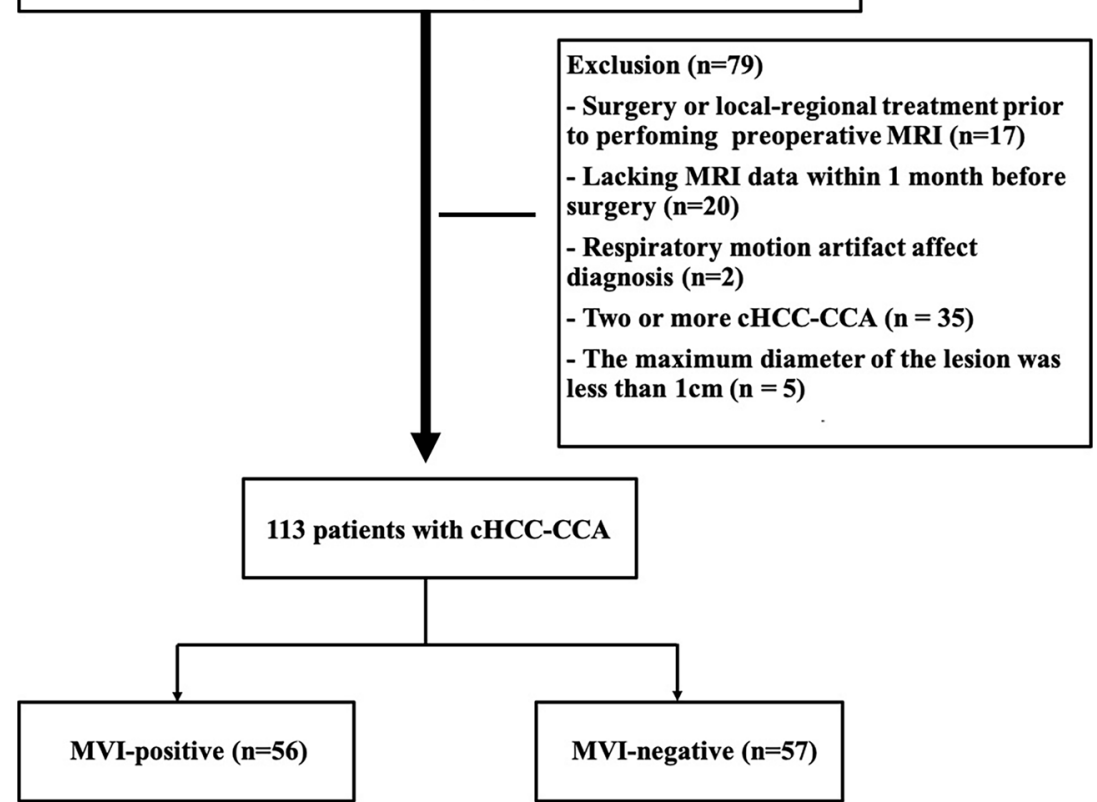

1 month before surgery $(n=20)$; poor MRI quality, including respiratory motion artifact effects $(n=2)$; two or more lesions of cHCC-CCA in the same liver $(n=35)$; and the maximum diameter of the lesion was less than $1 \mathrm{~cm}(n=5)$. Finally, 113 patients with cHCC-CCA were enrolled in this study (Fig. 1).

\section{MRI acquisition}

All patients were examined with a 24-channel 1.5-T magnetic scanner (uMR 560; United Imaging Healthcare). Routine plain-scan liver protocols consisted of a transverse T2weighted breath-hold fat-suppressed fast spin-echo sequence, T1-weighted breath-hold in-phase and opposed-phase gradient echo sequence, and free-breath diffusion-weighted imaging (DWI) with a transverse single-shot spin-echo planar sequence ( $b$ value, 0,50 , and $500 \mathrm{~s} / \mathrm{mm}^{2}$ ). Dynamic imaging was performed with a breath-hold T1-weighted 3-dimensional fat-suppressed quick spoiled gradient echo sequence before the intravenous administration of gadoliniumdiethylenetriamine pentaacetic acid (Gd-DTPA) (Magnevist; Bayer HealthCare). Gd-DTPA was administered at a dose of $0.1 \mathrm{mmol} / \mathrm{kg}$ at a rate of $2 \mathrm{ml} / \mathrm{s}$, followed by a $20-\mathrm{ml}$ saline flush using a power injector (Spectris; Medrad). The arterial phase acquisition was triggered automatically by monitoring when the contrast media reached the ascending aorta. For subsequent acquisitions, dynamic T1-weighted MRI at 70$90 \mathrm{~s}$ (the portal venous phase) and 160-180 s (the delay phase) was performed. The detailed parameters of each acquisition sequence are shown in Table 1.

\section{Imaging analysis}

All MRI scans were retrospectively analyzed together using a picture archiving and communication system (PACS;

Table 1 Parameters of T1-weighted imaging, T2-weighted imaging and diffusion-weighted imaging

\begin{tabular}{|c|c|c|c|c|}
\hline Parameter & FS-T2-weighted & T1-weighted IP and OP imaging & FS-T1-weighted quick3D BH & DWI \\
\hline Repetition time (ms) & 2693 & 115.8 & 4.4 & 2807 \\
\hline Echo time (ms) & 85.6 & 4.7 and 2.2 & 2.2 & 75.7 \\
\hline Matrix size & $201 \times 288$ & $230 \times 288$ & $192 \times 256$ & $115 \times 128$ \\
\hline Field of view $\left(\mathrm{mm}^{2}\right)$ & $380 \times 360$ & $400 \times 280$ & $400 \times 280$ & $380 \times 300$ \\
\hline Slice thickness (mm) & 6.0 & 6.0 & 3.0 & 6.0 \\
\hline Slice gap (mm) & 1.2 & 1.2 & 0 & 1.2 \\
\hline
\end{tabular}

$F S$ fat-suppression, $I P$ in-phase, $O P$ opposed-phase, $3 D$ three dimensional, $B H$ breath-hold, $D W I$ diffusion-weighted imaging 
Table 2 Clinical characteristics of cHCC-CCA according to MVI

\begin{tabular}{|c|c|c|c|}
\hline Clinical parameters & $\begin{array}{l}\text { MVI-positive } \\
(n=56)\end{array}$ & $\begin{array}{l}\text { MVI-negative } \\
(n=57)\end{array}$ & $p$ value \\
\hline Age (years) ${ }^{a}$ & $56.9 \pm 11.4$ & $52.7 \pm 11.6$ & 0.0561 \\
\hline Sex (male:female) & $37: 19$ & $40: 17$ & 0.640 \\
\hline Largest diameter $(\mathrm{cm})^{\mathrm{a}}$ & $5.4 \pm 3.2$ & $3.7 \pm 1.9$ & 0.0009 \\
\hline $1-5 \mathrm{~cm}$ & $26(46.4)$ & $41(71.9)$ & 0.006 \\
\hline$>5 \mathrm{~cm}$ & $30(53.6)$ & $16(28.1)$ & \\
\hline Etiology $^{\dagger}$ & & & 0.290 \\
\hline Hepatitis B virus & $42(75.0)$ & $48(84.2)$ & \\
\hline Hepatitis $\mathrm{C}$ virus & $1(1.8)$ & $0(0)$ & \\
\hline None or other & $13(23.2)$ & $9(15.8)$ & \\
\hline \multicolumn{4}{|l|}{ Liver functional parameters } \\
\hline Total bilirubin $>20(\mu \mathrm{mol} / \mathrm{L})$ & $8(14.3)$ & $9(15.8)$ & 0.823 \\
\hline Direct bilirubin $>7(\mu \mathrm{mol} / \mathrm{L})$ & $7(12.5)$ & $12(21.1)$ & 0.224 \\
\hline Alanine aminotransferase $>40$ (IU/L) & $15(26.8)$ & $18(31.6)$ & 0.575 \\
\hline Aspartate aminotransferase $>40$ (IU/L) & $13(23.2)$ & $10(17.5)$ & 0.454 \\
\hline$\gamma$-Glutamyltranspeptidase $>60$ (IU/L) & $26(46.4)$ & $28(49.1)$ & 0.774 \\
\hline Albumin $<35(\mathrm{~g} / \mathrm{L})^{\dagger}$ & $3(5.4)$ & $2(3.5)$ & 0.679 \\
\hline \multicolumn{4}{|l|}{ Tumor markers } \\
\hline Alpha-fetoprotein $\geq 20$ and $<400(\mathrm{ng} / \mathrm{ml})$ & $21(37.5)$ & $22(38.6)$ & 0.796 \\
\hline Alpha-fetoprotein $\geq 400$ (ng/ml) & $18(32.1)$ & $8(14.0)$ & 0.022 \\
\hline Cancer antigen 19-9>37 (U/ml) & $13(23.2)$ & $14(24.6)$ & 0.867 \\
\hline Carcinoembryonic antigen $>5(\mathrm{ng} / \mathrm{ml})$ & $12(21.4)$ & $8(14.0)$ & 0.303 \\
\hline
\end{tabular}

Data are numbers of patients (percentage), unless otherwise specified

${ }^{\mathrm{a}}$ Data are means \pm standard deviations

${ }^{\dagger}$ Data were compared using the Fisher's exact test. The ages were compared using an independent sample $t$ test. Excepted where indicated, data were compared using the $\chi^{2}$ test
Pathspeed, GE Medical Systems Integrated Imaging Solutions) by two radiologists (X.L.W. and C.Y., with 7 and 13 years of experience in abdominal imaging, respectively). Both radiologists were aware that all patients had $\mathrm{cHCC}-\mathrm{CCA}$ but were blinded to other clinical data, laboratory tests, and pathology results. A third experienced abdominal radiologist (K.X.) with more than 30 years of experience was invited to resolve any disagreements between the two observers.

\section{Qualitative analysis}

The following qualitative imaging parameters of the lesions were evaluated on the plain scan: (a) shape of the tumors (globular, lobulated or irregular); (b) contour (smooth or nonsmooth margin); (c) homogeneous or heterogeneous on T2WI; (d) tumor location (right, left, both, or other liver lobe); (e) hemorrhage/hemosiderin; (f) intratumoral fat deposition; (g) necrosis; (h) upper abdominal lymphadenopathy (lymph nodes $>1 \mathrm{~cm}$ on the short axis); (i) peritumoral bile duct dilatation; and (j) hepatic capsular retraction. Dynamic enhancement characteristics were as follows: (A) arterial phase-(a) hypervascularity or nonhypervascularity; (b) homogeneity or heterogeneity enhancement; and (c) peritumoral enhancement patterns (assessed as detectable enhancing portion adjacent to the tumor border [wedge shaped], an extensive enhancement surrounding the tumor border [irregular shaped], or absent); (B) portal venous phase-(d) washout (nonperipheral washout or peripheral washout) and (e) enhancing capsule (complete, incomplete, or absent); (C) in the targetoid mass - ( $\mathrm{f}$ ) rim-APHE; ( $\mathrm{g}$ ) peripheral washout; (h) progressive central enhancement; and (i) targetoid diffusion restriction. In addition, all the lesions were categorized based on the LI-RADS v2018, LR-M (definitely or probably malignant, not HCC specific, including rim APHE, peripheral washout, and delayed and progressive concentric enhancement). Threshold growth was excluded because many patients had only one preoperative MRI examination.

\section{Clinical data and MVI pathological evaluation}

The following clinical data were collected from the medical records: (a) demographic characteristics (age, sex); (b) etiology (hepatitis $\mathrm{B}$ or $\mathrm{C}$ virus infection, schistosomiasis, average daily alcohol consumption $>100 \mathrm{~g} /$ day, without obvious 
Table 3 Comparison of qualitative data obtained on MRI features stratified by MVI status

\begin{tabular}{|c|c|c|c|}
\hline MRI features & $\begin{array}{l}\text { MVI-positive } \\
(n=56)\end{array}$ & $\begin{array}{l}\text { MVI-negative } \\
(\mathrm{n}=57)\end{array}$ & $p$ value \\
\hline Shape $^{\dagger}$ & & & 0.025 \\
\hline Irregular & $7(12.5)$ & $3(5.3)$ & \\
\hline Lobulated & $38(67.8)$ & $30(52.6)$ & \\
\hline Globular & $11(19.6)$ & $24(42.1)$ & \\
\hline Contour smooth & $14(25.0)$ & $20(35.1)$ & 0.242 \\
\hline Homogeneity $\mathrm{T} 2$ & $34(60.1)$ & $39(68.4)$ & 0.392 \\
\hline Hemorrhage / hemosiderin & $16(28.6)$ & $7(12.3)$ & 0.032 \\
\hline Fat deposition ${ }^{\dagger}$ & $3(5.4)$ & $13(22.8)$ & 0.013 \\
\hline Necrosis & $20(35.7)$ & $14(24.6)$ & 0.196 \\
\hline Upper abdominal lymphadenopathy & $22(39.3)$ & $10(17.5)$ & 0.010 \\
\hline Location $^{\dagger}$ & & & 0.953 \\
\hline Right liver lobe & $40(71.4)$ & $41(71.9)$ & \\
\hline Left live lobe & $13(23.2)$ & $12(21.1)$ & \\
\hline Caudate lobe or border area & $3(5.4)$ & $4(7.0)$ & \\
\hline Arterial phase hyperenhancement & $50(89.2)$ & $56(98.2)$ & 0.061 \\
\hline Arterial phase homogeneity enhancement ${ }^{\dagger}$ & $2(3.6)$ & $9(15.8)$ & 0.053 \\
\hline Arterial phase peritumoral enhancement & & & $<0.001$ \\
\hline Absent & $13(23.2)$ & $39(69.4)$ & \\
\hline Wedge shaped & $19(33.9)$ & $9(15.8)$ & \\
\hline Irregular & $24(42.9)$ & $9(15.8)$ & \\
\hline Washout at portal venous phase & $28(50.0)$ & $30(52.6)$ & 0.780 \\
\hline \multicolumn{4}{|l|}{ Enhancing capsule } \\
\hline Complete & $11(19.6)$ & $14(24.6)$ & 0.275 \\
\hline Incomplete & $12(21.4)$ & $6(10.5)$ & \\
\hline Absent & $33(58.9)$ & $37(64.9)$ & \\
\hline \multicolumn{4}{|l|}{ Targetoid mass } \\
\hline Rim arterial phase hyperenhancement & $27(48.2)$ & $33(57.9)$ & 0.303 \\
\hline Peripheral washout & $12(21.4)$ & $18(31.6)$ & 0.222 \\
\hline Progressive central enhancement & $38(67.8)$ & $43(75.4)$ & 0.371 \\
\hline Targetoid diffusion restriction ${ }^{\dagger}$ & $1(1.8)$ & $5(8.8)$ & 0.206 \\
\hline Peritumoral bile duct dilatation & $14(25.0)$ & $6(10.5)$ & 0.044 \\
\hline Surface retraction ${ }^{\dagger}$ & $1(1.8)$ & $1(1.8)$ & 1.000 \\
\hline LI-RADS categorization $^{\dagger}$ & & & 0.819 \\
\hline LR-4 & $1(1.8)$ & $1(1.8)$ & \\
\hline LR-5 & $12(21.4)$ & $10(17.5)$ & \\
\hline LR-M & $43(76.8)$ & $46(80.7)$ & \\
\hline LR-TIV $^{\dagger}$ & $7(12.5)$ & $2(3.5)$ & 0.124 \\
\hline
\end{tabular}

The data are presented as the number $(\%)$ of patients

$\dagger$ Data were compared using the Fisher's exact test. LR-4 probably HCC, LR-5 definitely HCC, LR-M definitely or probably malignant, not HCC specific, LR-TIV tumor in vein causes); (c) largest tumor diameter (divided into the $1-5 \mathrm{~cm}$ group and the $>5 \mathrm{~cm}$ group); (d) liver functional parameters (alanine aminotransferase [ALT], aspartate aminotransaminase [AST], $\gamma$-glutamyltranspeptidase [GGT], albumin [ALB], total bilirubin [TB], and direct bilirubin [DB]; and (e) tumor biomarkers ( $\alpha$-fetoprotein [AFP], carcinoembryonic antigen [CEA], and cancer antigen 19-9 [CA19-9]).

The pathological characteristics of the hepatectomy specimens were evaluated by a team of experienced pathologists (each individual had more than 12 years of experience in reading histopathological slices), who were blinded to the MRI and clinical results. MVI was defined as tumor cells within a vascular space lined by endothelium located in the periphery of the tumor at the tumor and liver parenchyma interface that was visible only by microscopy. The enrolled patients were divided into two groups (MVI-positive and MVI-negative) based on pathological characteristics.

\section{Follow-up RFS after surgery}

All of the enrolled 113 patients with $\mathrm{cHCC}-\mathrm{CCAs}$ underwent R0 liver resection (no residual tumor) within 30 days after the first MRI examination, with the surgical techniques and perioperative management the same as in previous reports [4]. Follow-up for RFS consisted of chest radiography, laboratory tests including serum AFP or protein induced by vitamin $\mathrm{k}$ absence or antagonist-II (PIVKA-II), and abdominal MRI at 1 month after surgery; if there was no recurrence, the patient was reexamined every 2-3 months. If only the level of a tumor marker increased without any radiographic evidence of a new 
Fig. 2 Images in a 57-year-old man with $\mathrm{cHCC}-\mathrm{CCA}$

categorized as LR-M with MVI. a Axial arterial phase image shows a $3.5-\mathrm{cm}$ rim hyperenhancement lesion (arrow) in segment IV of the liver. b Portal venous phase image shows continuous peripheral enhancement and progressive central enhancement (arrow). c Delay phase image shows a further progressive central enhancement appearance (arrow). d Diffusion-weighted image shows targetoid appearance $\left(b=500 \mathrm{~s} / \mathrm{mm}^{2}\right)$ with peripheral hyperintensity and central relatively hypointensity (arrow)
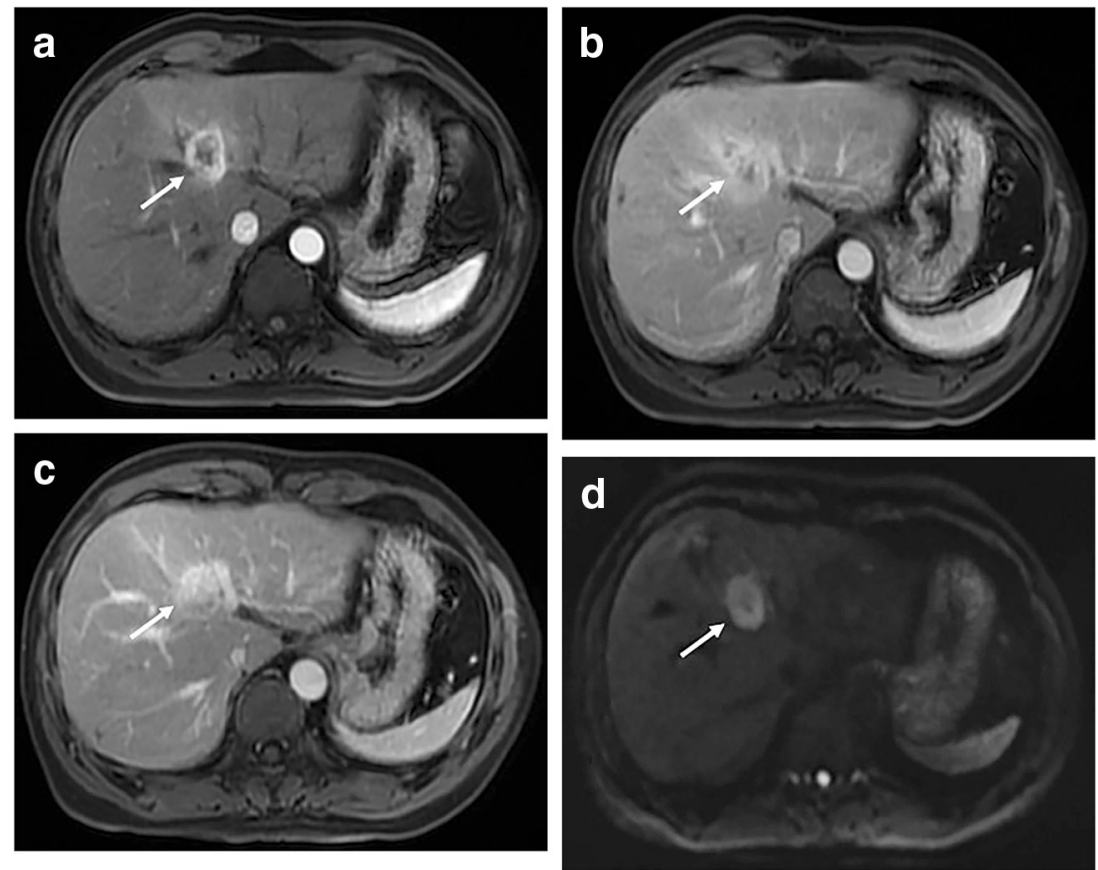

tumor, follow-up was continuous until a tumor presented on imaging, at which point the time of recurrence was recorded.

\section{Statistical analysis}

All statistical analyses were performed using SPSS 20.0 and MedCalc software (version 15.0). Normally distributed data are expressed as the means \pm standard deviations, and comparisons between the two groups were performed using independent sample $t$ tests. The data with skewed distributions are expressed as the medians $(25 \%, 75 \%)$, and comparisons between the two groups were performed using rank sum tests. Categorical variables are reported as the numbers of cases and percentages, and $\chi^{2}$ or Fisher's exact tests were used. Comparisons between groups of categorical variables were performed by one-way analysis of variance. Parameters were analyzed using univariate and multivariate logistic regression to determine whether they were independent risk factors predicting MVI (the univariate analysis was performed first, and only those parameters found to have statistical significance were used in the stepwise multivariate logistic regression). A $p$ value less than 0.05 indicated a significant difference. The odds ratio (OR) and $95 \%$ confidence interval (CI) were calculated. The sensitivity, specificity, accuracy, positive predictive value (PPV), and negative predictive value (NPV) were calculated for each significant finding and combinations of significant findings on multivariate logistic regression with regard to predicting MVI. The RFS after hepatectomy in two groups were estimated using Kaplan-Meier survival curves and compared using the log-rank test.

\section{Results}

\section{Patient clinical and MR characteristics}

The comparisons of patient clinical characteristics stratified by the MVI status and data are detailed in Table 2. The results revealed MVI-positive lesions in 56 patients $(49.6 \%)$ and MVI-negative lesions in 57 patients $(50.4 \%)$. There were significant differences in the tumor size $>5 \mathrm{~cm}$ and the level of serum AFP $\geq 400 \mathrm{ng} / \mathrm{ml}$ between MVI-positive and MVInegative groups ( $p=0.006$ and $p=0.022$, respectively), but when the serum level of AFP was between 20 and $400 \mathrm{ng} / \mathrm{ml}$, there was no significant differences between the two groups. No significant differences $(p>0.05)$ were found in age, sex, etiology, liver functional parameters, or the levels of CA19-9 and CEA between the two groups (Table 2).

Among the recorded MRI characteristics (Table 3), tumor shape $(p=0.025)$, hemorrhage/hemosiderin $(p=0.032)$, intratumoral fat deposition $(p=0.013)$, upper abdominal lymphadenopathy $(p=0.010)$, the arterial phase peritumoral enhancement pattern $(p<0.001)$, and peritumoral bile duct dilatation $(p=0.044)$ were significantly associated with MVI. Most (89/ $113,78.8 \%$ ) of the cHCC-CCA could be properly categorized as LR-M (Fig. 2), and no significant difference in MVI was found based on the LI-RADS category $(p=0.819)$. Other features did not differ between the two groups (Table 3 ).

\section{Univariate and multivariate analyses}

Univariate logistic regression analysis showed that there were eight risk factors that were significantly related to the MVI of 
Table 4 Univariate and multivariate analyses of risk factors for the MVI of cHCCCCA

\begin{tabular}{|c|c|c|c|c|}
\hline \multirow[t]{2}{*}{ Risk factor } & \multicolumn{2}{|l|}{ Univariate analysis } & \multicolumn{2}{|l|}{ Multivariate analysis } \\
\hline & Odds ratio $(95 \% \mathrm{CI})$ & $p$ value & Odds ratio $(95 \% \mathrm{CI})$ & $p$ value \\
\hline Age (years) ${ }^{a}$ & $0.968(0.936-1.001)$ & 0.060 & $\ldots$ & $\ldots$ \\
\hline Sex (male:female) & $0.828(0.375-1.828)$ & 0.640 & $\ldots$ & $\ldots$ \\
\hline Largest diameter $(\mathrm{cm})$ & $0.772(0.655-0.908)$ & 0.002 & $1.010(0.788-1.94)$ & 0.937 \\
\hline Alpha-fetoprotein $\geq 400(\mathrm{ng} / \mathrm{ml})$ & $0.533(0.324-0.876)$ & 0.013 & $0.523(0.282-0.971)$ & 0.040 \\
\hline Cancer antigen 19-9> $37(\mathrm{U} / \mathrm{ml})$ & $1.077(0.453-2.558)$ & 0.060 & $\ldots$ & $\ldots$ \\
\hline Carcinoembryonic antigen $>5(\mathrm{ng} / \mathrm{ml})$ & $0.490(0.187-1.282)$ & 0.146 & $\ldots$ & $\ldots$ \\
\hline Shape & $\ldots$ & $\ldots$ & $\ldots$ & $\ldots$ \\
\hline Irregular & $0.387(0.199-0.753)$ & 0.005 & $0.718(0.293-1.758)$ & 0.469 \\
\hline Lobulated* & $\ldots$ & $\ldots$ & $\ldots$ & $\ldots$ \\
\hline Globular* & $\ldots$ & $\ldots$ & $\ldots$ & $\ldots$ \\
\hline Contour smooth & $1.621(0.719-3.658)$ & 0.244 & $\ldots$ & $\ldots$ \\
\hline Hemorrhage/hemosiderin & $0.350(0.131-0.933)$ & 0.036 & $0.910(0.252-3.280)$ & 0.885 \\
\hline Fat deposition & $5.220(1.398-19.490)$ & 0.014 & $14.368(2.749-75.098)$ & 0.002 \\
\hline Necrosis & $0.972(0.453-2.085)$ & 0.942 & $\ldots$ & $\ldots$ \\
\hline Upper abdominal lymphadenopathy & $0.328(0.138-0.783)$ & 0.012 & $0.358(0.118-1.087)$ & 0.070 \\
\hline Arterial phase homogeneity enhancement & $5.062(1.041-24-596)$ & 0.044 & $1.932(0.295-12.643)$ & 0.492 \\
\hline Arterial phase peritumoral enhancement & $\ldots$ & $\ldots$ & $\ldots$ & $\ldots$ \\
\hline Absent* & $\ldots$ & $\ldots$ & $\ldots$ & $\ldots$ \\
\hline Wedge shaped* & $\ldots$ & $\ldots$ & $\ldots$ & .. \\
\hline Irregular & $0.332(0.201-0.550)$ & $<0.001$ & $0.322(0.164-0.631)$ & 0.001 \\
\hline Enhancing capsule & $0.675(0.320-1.426)$ & 0.303 & $\ldots$ & $\ldots$ \\
\hline Targetoid mass & $\ldots$ & $\ldots$ & $\ldots$ & $\ldots$ \\
\hline Rim arterial phase hyperenhancement & $1.477(0.703-3.103)$ & 0.303 & $\ldots$ & $\ldots$ \\
\hline Peripheral washout & $1.692(0.724-3.952)$ & 0.224 & $\ldots$ & $\ldots$ \\
\hline Progressive central enhancement & $1.455(0.639-3.315)$ & 0.373 & $\ldots$ & $\ldots$ \\
\hline Targetoid diffusion restriction & $5.288(0.598-46.794)$ & 0.134 & $\ldots$ & $\ldots$ \\
\hline Peritumoral bile duct dilatation & $0.353(0.125-0.998)$ & 0.053 & $\ldots$ & $\ldots$ \\
\hline Surface retraction & $0.982(0.060-16.097)$ & 0.990 & $\ldots$ & $\ldots$ \\
\hline LR-M & $1.205(0.539-2.690)$ & 0.650 & $\ldots$ & $\ldots$ \\
\hline
\end{tabular}

${ }^{\mathrm{a}}$ Data are the means \pm standard deviations

*Data were used as the reference variable. LR-M definitely or probably malignant, not HCC specific
cHCC-CCA (Table 4). A larger tumor size $(p=0.002)$, a higher serum level of AFP ( $p=0.013)$, an irregular shape $(p=0.005)$, hemorrhage/hemosiderin $(p=0.036)$, intratumoral fat deposition $(p=0.014)$, upper abdominal lymphadenopathy $(p=0.012)$, arterial phase homogeneity enhancement $(p=0.044)$, and irregular arterial peritumoral enhancement $(p<0.001)$ were associated with MVI. These parameters were analyzed using multivariate logistic regression.
Table 5 Diagnostic performance of independent risk factors for the prediction of MVI in cHCC-CCA

\begin{tabular}{llllll}
\hline Factors & Sensitivity & Specificity & Accuracy & PPV & NPV \\
\hline $\begin{array}{l}\text { Alpha-fetoprotein } \geq 400 \\
\quad\left(\mathrm{ng} / \mathrm{ml}^{\mathrm{a}}\right.\end{array}$ & $32.1(18 / 56)$ & $86.0(49 / 57)$ & $59.3(67 / 113)$ & $69.2(18 / 26)$ & $56.3(49 / 87)$ \\
$\begin{array}{l}\text { Without intratumoral fat } \\
\quad \text { deposition }\end{array}$ & $94.6(53 / 56)$ & $22.8(13 / 57)$ & $58.4(66 / 113)$ & $54.6(53 / 97)$ & $81.3(13 / 16)$ \\
$\begin{array}{l}\text { Irregular peritumoral } \\
\quad \text { enhancement }^{\mathrm{c}}\end{array}$ & $42.9(24 / 56)$ & $84.2(48 / 57)$ & $63.7(72 / 113)$ & $72.7(24 / 33)$ & $60.0(48 / 80)$ \\
$\begin{array}{l}\text { Combination of a and b } \\
\text { Combination of b and c }\end{array}$ & $32.1(18 / 56)$ & $87.7(50 / 57)$ & $60.2(68 / 113)$ & $72.0(18 / 25)$ & $56.8(50 / 88)$ \\
$\begin{array}{l}\text { Combination of a and c } \\
\text { Combination of all three } \\
\text { factors }\end{array}$ & $17.9(10 / 56)$ & $96.5(55 / 57)$ & $57.5(65 / 113)$ & $83.3(10 / 12)$ & $54.5(55 / 101)$ \\
& $12.5(7 / 56)$ & $98.2(56 / 57)$ & $55.8(63 / 113)$ & $87.5(7 / 8)$ & $53.3(56 / 105)$ \\
\hline
\end{tabular}

Data are presented as percentages. Data in parentheses are the numbers of subjects used to calculate the percentage

${ }^{\text {a }}$ Alpha-fetoprotein $\geq 400(\mathrm{ng} / \mathrm{ml})$

${ }^{\mathrm{b}}$ Without intratumoral fat deposition

${ }^{\mathrm{c}}$ Irregular peritumoral enhancement

$P P V$ positive predictive value, $N P V$ negative predictive value 
Fig. 3 Images in a 58-year-old woman with cHCC-CCA with MVI; her serum level of AFP was $1885 \mathrm{ng} / \mathrm{ml}$. a In-phase MR image shows a $3.0-\mathrm{cm}$ hypointense irregular mass (arrow) in segment VI of the liver. b On the opposed-phase image, there was no obvious signal drop in the lesion (arrow), indicating the absence of an unambiguous fatty-containing lesion. c Axial arterial phase image shows a hypervascular mass with irregular peritumoral enhancement (arrow). d Portal venous phase image shows nonsmooth tumor margin and peritumoral slight hypointensity (arrow)
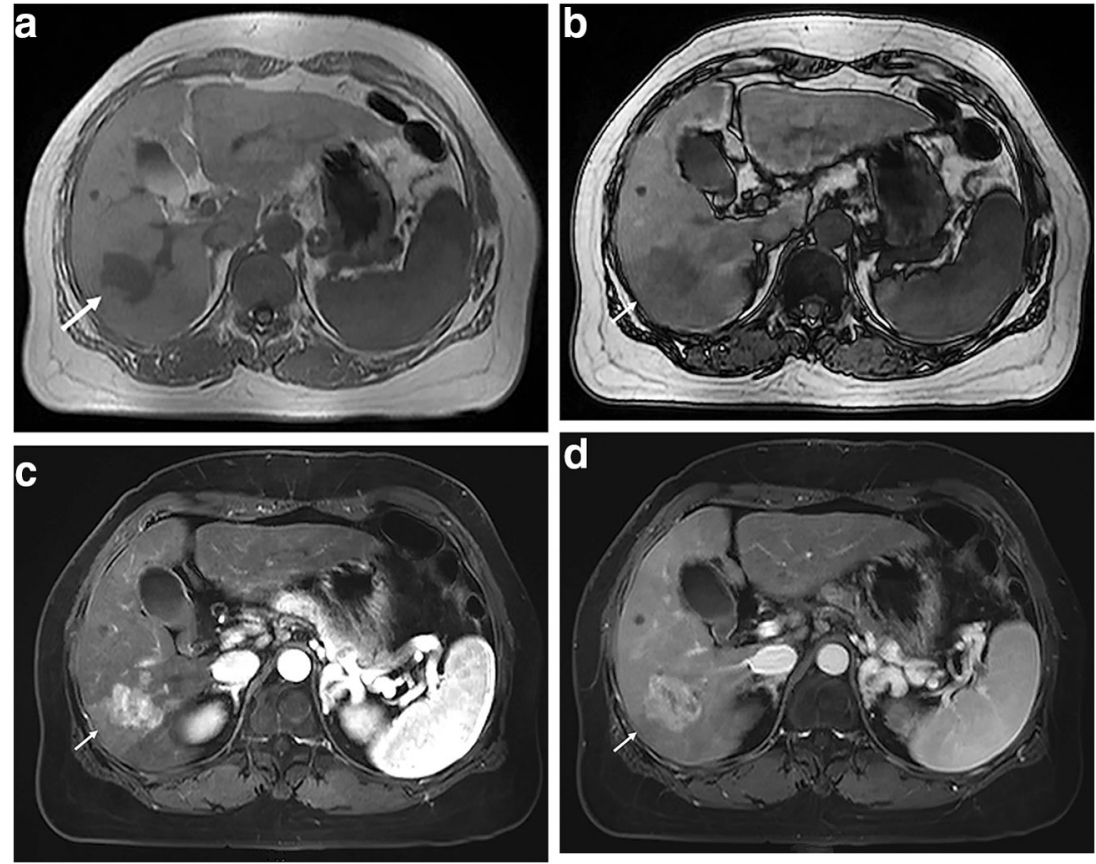

Higher serum levels of AFP (odds ratio [OR], 0.523; 95\% confidence interval [CI], 0.282-0.971; $p=0.040)$, intratumoral fat deposition (OR, 14.368; 95\% CI, 2.749$75.098 ; p=0.002)$, and irregular arterial peritumoral enhancement (OR, 0.322; 95\% CI, 0.164-0.631; $p=0.001)$ were independent variables associated with the MVI of cHCC-CCA.

The sensitivity, specificity, accuracy, PPV, and NPV for the prediction of MVI by the three significant factors and their combination are shown in Table 5 . When all three factors were combined (Fig. 3), the specificity was $98.2 \%$ (56/57), and the sensitivity was $12.5 \%(7 / 56)$.

\section{RFS outcomes after surgery}

All 113 patients with cHCC-CCAs received R0 liver resection (no residual tumor) within 30 days after the first MRI examination. After hepatectomy, patients with MVI of cHCC-CCA had a median RFS of 10.8 months (range 1-25 months), while those without MVI had a median RFS of 25.4 months (range $1-40$ months), and the early recurrence rates ( $<2$ years) were estimated to be $83.9 \%(47 / 56)$ and $49.1 \%$ (28/57), respectively. There was a significant difference in RFS between patients with MVI-positive and MVI-negative tumors (hazard ratio [HR], 0.402; 95\% CI, 0.189-0.854, $p=0.013)$. KaplanMeier survival curves were generated (Fig. 4).

\section{Discussion}

Our results illustrated that a higher serum level of AFP and irregular arterial phase peritumoral enhancement may indicate a higher risk of the MVI of cHCC-CCA, while intratumoral fat detected on MRI suggests a lower risk. Combining these three findings for the prediction of MVI resulted in specificity greater than $98 \%$. In addition, cHCC-CCAs with MVI may have worse surgical outcomes with regard to early recurrence than those without MVI.

Previously, a few studies reported that a higher serum level of AFP was one of the independent risk factors associated with MVI in HCC [32, 33] and ICC [34] patients. Our findings also showed that a higher serum level of AFP was an independent predictor of MVI in cHCC-CCA, but when the serum level of AFP was between 20 and $400 \mathrm{ng} / \mathrm{ml}$, there were no significant differences in MVI. Furthermore, some studies have suggested that the clinical characteristics of cHCC-CCA are similar to those of HCC; for example, the majority of cHCC-CCAs occur against a background of positive hepatitis $\mathrm{B}$ serology and cirrhosis, and the patients are predominately male $[28,35]$. Our results were consistent with these studies. In this study, the patients had a sex ratio (male: female) of $77: 36$, and most patients $(79.6 \%)$ had been infected with the hepatitis B virus; however, no significant differences in age, sex, or etiology were found regard to in MVI.

Intratumoral fat deposition was an additional significant factor for predicting a lower risk of the MVI of cHCC-CCA in our study, which was consistent with some reports. Min et al [36] described that intratumoral fat was one of the independent variables for suggesting a lower risk of the MVI of HCC. A few studies have suggested that intratumoral fatty changes are associated with favorable tumor grades on histologic examination and a lower likelihood of MVI; therefore, fat-containing lesions may predict a more favorable prognosis 
Fig. 4 Kaplan-Meier survival curve for the recurrence-free survival of patients with $\mathrm{cHCC}$ CCAs that were MVI-positive and MVI-negative ( $\mathrm{HR}=0.402$; 95\% CI, 0.189-0.854, $p=0.013$ ). cHCC-CCA, combined hepatocellularcholangiocarcinoma; MVI, microvascular invasion; HR, hazard ratio; $\mathrm{CI}$, confidence interval

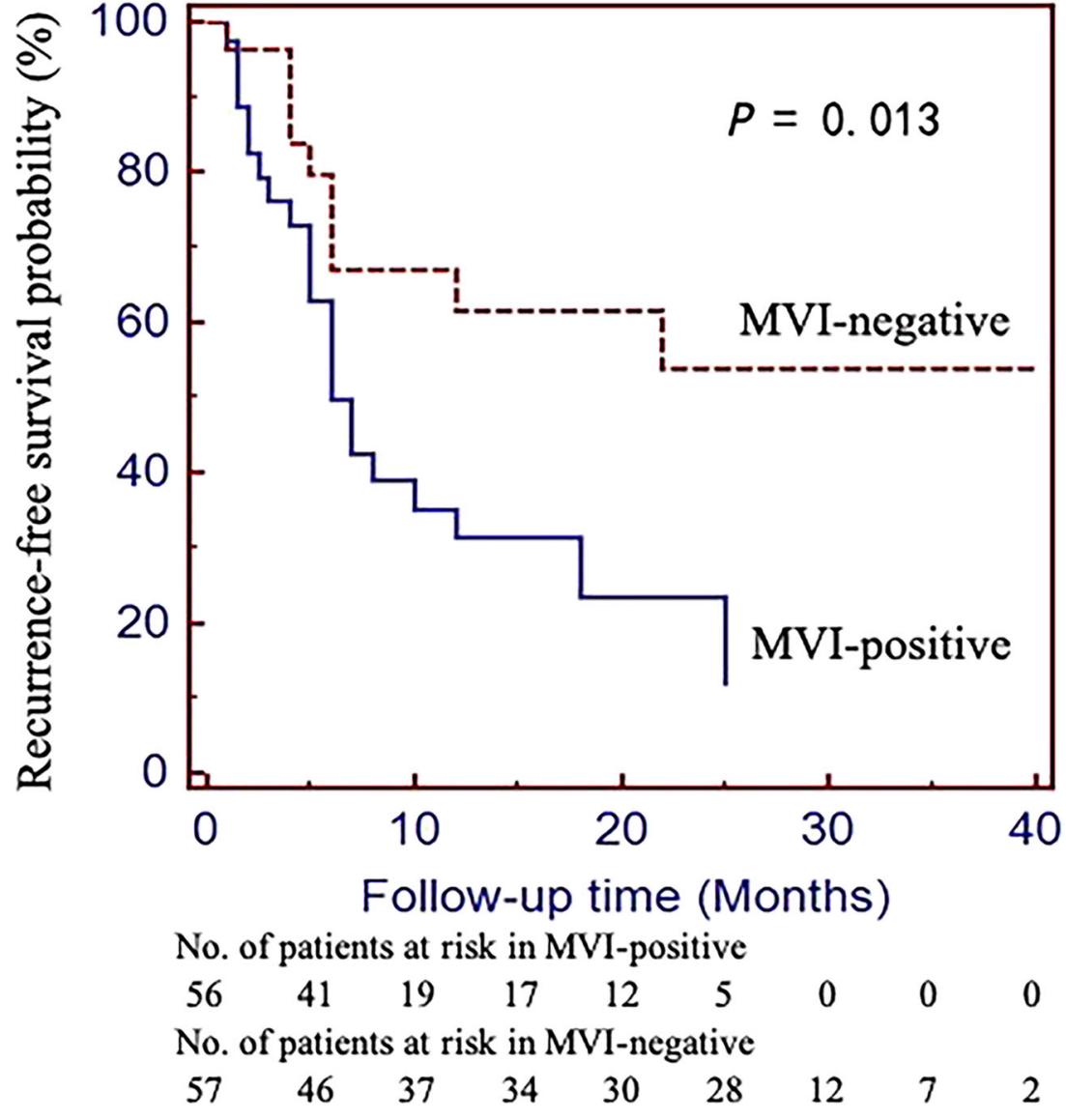

than non-fat-containing lesions [36, 37]. Moreover, as is well known, fatty changes in HCC are associated with ischemia, which may be related to a reduced normal portal vein blood supply [38]. Increased intratumoral fat may indicate less aggressive $\mathrm{HCC}$, as evidenced by the fact that $\mathrm{HCC}$ with diffuse fat tends to grow slowly. Because our sample size for fatcontaining cHCC-CCA with MVI was relatively small, the relationship between intratumoral fat and the prognosis of cHCC-CCA remains to be further studied.

Our study also showed that irregular arterial phase peritumoral enhancement was a significant MRI finding predicting the MVI of cHCC-CCA. Many reports [18, 20, 39] have shown that arterial peritumoral enhancement is an independent predictive factor of MVI in HCC. To date, few studies have described the relationship between peritumoral enhancement of cHCC-CCA and MVI. The mechanism of hemodynamic changes in this type of MRI feature is interpreted as a decrease in or disappearance of portal blood flow due to tumor thrombosis in the microportal branch around the tumor, resulting in compensatory hepatic arterial hyperperfusion [40]. In addition, although previous studies have reported that a large tumor size could be considered a major predictor of HCC with MVI [36], it has not always been considered an independent predictor of the MVI of HCC [18, 19]. In this study, tumor size, tumor shape, intratumoral hemorrhage, upper abdominal lymphadenopathy, and arterial phase heterogeneity enhancement were important risk factors for the MVI of cHCC-CCA in univariate analysis, but they were not independent factors predicting MVI.

It has been reported that MVI is one of the most important prognostic factor for the early recurrence of HCC after hepatic resection or radiofrequency ablation [20, 39]; we also found that cHCC-CCAs with MVI may have worse surgical outcomes with regard to early recurrence than those without MVI. Recent studies [30] have reported that patients with cHCC-CCAs in the LR$\mathrm{M}$ category had a higher early recurrence rate $(\leq$ 6 months) than those with cHCC-CCAs in the LR-5/4 categories. While there was no significant difference in RFS, cHCC-CCAs mimicking HCCs on imaging (LR-5/ 4) may have improved surgical outcomes. Unlike this study, a substantial proportion of $\mathrm{cHCC}-\mathrm{CCAs}$ were categorized as LR-M $(78.8 \%, 89 / 113)$ in our study; nevertheless, no significant difference in the MVI of cHCCCCA was found based on the LI-RADS categories.

This study has several limitations. First, because this research was a single-center and retrospective study, there might have been selection bias. Second, tumor size and the number of lesions were confined to larger than $1 \mathrm{~cm}$ in maximum diameter and a single mass in this study; therefore, the 
conclusions cannot be generalized to other size lesions or two or more lesions. Third, the data for overall survival (OS) were not available; thus, the relationship between the MVI of cHCC-CCA and OS requires further research in the future. Fourth, in this study, Gd-DTPA was used as a contrast agent for MRI; therefore, further research is warranted on gadoxetic acid-enhanced MRI for the identification of the MVI of cHCC-CCA. Fifth, our sample size for fat-containing cHCCCCA with MVI was relatively small which resulted in a low diagnostic sensitivity when all the three parameters were combined; therefore, more patients needed to be enrolled to clarify the diagnostic efficacy. Finally, in our study, cHCC-CCA was assessed only as either MVI-positive or MVI-negative. In a recent study [41], MVI was further categorized into different grades based on the number of vessels invaded. Further study is needed to assess the relationship between preoperative clinical or MRI findings and different grades of MVI of cHCCCCA.

In summary, the proportion of MVI-positive patients accounts for approximately half of all cHCC-CCA patients. Higher serum levels of AFP and irregular arterial peritumoral enhancement were independent variables associated with the MVI of cHCC-CCA, while fat deposition might be a protective factor. In addition, cHCC-CCA with MVI may have a higher early recurrence rate after surgery.

Funding information This study has received funding by the National Natural Science Foundation of China (grant number 91859107), the Shanghai Science and Technology Committee (grant number18DZ1930102), the Shanghai Science and Technology Committee (grant number 19411965500), the Zhongshan Hospital, Fudan University (grant number 2018ZSLC22), and the Shanghai Municipal Key Clinical Specialty (grant number W2019-018).

\section{Compliance with ethical standards}

Guarantor The scientific guarantor of this publication is Chun Yang.

Conflict of interest The authors of this manuscript declare no relationships with any companies whose products or services may be related to the subject matter of the article.

Statistics and Biometry No complex statistical methods were necessary for this paper.

Informed consent Written informed consent was waived by the Institutional Review Board.

Ethical approval This retrospective study performed at one institution was approved by the Institutional Review Board of Zhongshan Hospital of Fudan University.

\section{Methodology}

- Retrospective

- Diagnostic or prognostic study

- Performed at one institution
Open Access This article is licensed under a Creative Commons Attribution 4.0 International License, which permits use, sharing, adaptation, distribution and reproduction in any medium or format, as long as you give appropriate credit to the original author(s) and the source, provide a link to the Creative Commons licence, and indicate if changes were made. The images or other third party material in this article are included in the article's Creative Commons licence, unless indicated otherwise in a credit line to the material. If material is not included in the article's Creative Commons licence and your intended use is not permitted by statutory regulation or exceeds the permitted use, you will need to obtain permission directly from the copyright holder. To view a copy of this licence, visit http://creativecommons.org/licenses/by/4.0/.

\section{References}

1. European Association For The Study Of The Liver (2012) EASLEORTC clinical practice guidelines: management of hepatocellular carcinoma. J Hepatol 56:908-943

2. Jung DH, Hwang S, Hong SM et al (2016) Post-resection prognosis of combined hepatocellular carcinoma cholangiocarcinoma according to the 2010 WHO classification. World J Surg 41:1347-1357

3. Wang AQ, Zheng YC, Du J et al (2016) Combined hepatocellular cholangio- carcinoma: controversies to be addressed. World J Gastroenterol 22:4459-4465

4. Yin X, Zhang BH, Qiu SJ et al (2012) Combined hepatocellular carcinoma and cholangiocarcinoma: clinical features, treatment modalities, and prognosis. Ann Surg Oncol 19:2869-2876

5. Lee JH, Chung GE, Yu SJ et al (2011) Long-term prognosis of combined hepatocellular and cholangiocarcinoma after curative resection comparison with hepatocellular carcinoma and cholangiocarcinoma. J Clin Gastroenterol 45:69-75

6. Lee SD, Park SJ, Han SS et al (2014) Clinicopathological features and prognosis of combined hepatocellular carcinoma and cholangiocarcinoma after surgery. Hepatobiliary Pancreat Dis Int 13:594 601

7. Tang D, Nagano H, Nakamura M et al (2006) Clinical and pathological features of Allen's type C classification of resected combined hepatocellular and cholangio- carcinoma: a comparative study with hepatocellular carcinoma and cholangio-cellular carcinoma. J Gastrointest Surg 10:987-998

8. Groeschl RT, Turaga KK, Gamblin TC (2013) Transplantation versus resection for patients with combined hepatocellular carcinomacholangiocarcinoma. J Surg Oncol 107:608-612

9. Maganty K, Levi D, Moon J et a1 (2010) Combined hepatocellular carcinoma and intrahepatic cholangiocarcinoma: outcome after liver transplantation. Dig Dis Sci 55:3597-3601

10. Zhou YM, Sui CJ, Zhang XF, Li B, Yang JM (2017) Influence of cirrhosis on long-term prognosis after surgery in patients with combined hepatocellular-cholangio-carcinoma. BMC Gastroenterol 17: 25

11. Cong WM, Bu H, Chen J et al (2016) Practice guidelines for the pathological diagnosis of primary liver cancer: 2015 update. World J Gastroenterol 22:9279-9287

12. Mazzaferro V, Llovet JM, Miceli R et al (2009) Predicting survival after liver transplantation in patients with hepatocellular carcinoma beyond the Milan criteria: a retrospective, exploratory analysis. Lancet Oncol 10:35-43

13. Zhang XP, Wang K, Wei XB et al (2019) An eastern hepatobiliary surgery hospital microvascular invasion scoring system in predicting prognosis of patients with hepatocellular carcinoma and microvascular invasion after R0 liver resection: a large-scale, multicenter study. Oncologist 24:1-13 
14. Yuan SX, Yang F, Yang Y et al (2012) Long noncoding RNA associated with microvascular invasion in hepatocellular carcinoma promotes angiogenesis and serves as a predictor for hepatocellular carcinoma patients' poor recurrence-free survival after hepatectomy. Hepatology 56:2231-2241

15. Hu LS, Weiss M, Popescu I et al (2019) Impact of microvascular invasion on clinical outcomes after curative-intent resection for intrahepatic cholangio-carcinoma. J Surg Oncol 119:21-29

16. Wang C, Pang S, Si-Ma H et al (2019) Specific risk factors contributing to early and late recurrences of intrahepatic cholangiocarcinoma after curative resection. World J Surg Oncol 17:2

17. Feng ST, Jia Y, Liao B et al (2019) Preoperative prediction of microvascular invasion in hepatocellular cancer: a radiomics model using Gd-EOB-DTPA-enhanced MRI. Eur Radiol. https://doi.org/ 10.1007/s00330-018-5935-8

18. Wang WT, Yang L, Yang ZX et al (2018) Assessment of microvascular invasion of hepatocellular carcinoma with diffusion kurtosis imaging. Radiology 286:571-580

19. Wei Y, Huang Z, Tang H et al (2019) IVIM improves preoperative assessment of microvascular invasion in HCC. Eur Radiol. https:// doi.org/10.1007/s00330-019-06088-w

20. Lee S, Kim SH, Lee JE, Sinn DH, Park CK (2017) Preoperative gadoxetic acid-enhanced MRI for predicting microvascular invasion in patients with single hepatocellular carcinoma. J Hepatol 67:526-534

21. Zhu F, Yang F, Li J et al (2019) Incomplete tumor capsule on preoperative imaging reveals microvascular invasion in hepatocellular carcinoma: a systematic review and meta-analysis. Abdom Radiol (NY) 44:3049-3057

22. Zhou Y, Wang X, Xu C et al (2019) Mass-forming intrahepatic cholangiocarcinoma: can diffusion-weighted imaging predict microvascular invasion? J Magn Reson Imaging 50:315-324

23. Wells ML, Venkatesh SK, Chandan VS et al (2015) Biphenotypic hepatic tumors: imaging findings and review of literature. Abdom Imaging 40:2293-2305

24. Brunt E, Aishima S, Clavien PA et al (2018) cHCC-CCA: consensus terminology for primary liver carcinomas with both hepatocytic and cholangiocytic differentation. Hepatology 68:113-126

25. Fowler KJ, Sheybani A, Parker RA 3rd et al (2013) Combined hepatocellular and cholangiocarcinoma (biphenotypic) tumors: imaging features and diagnostic accuracy of contrast-enhanced CT and MRI. AJR Am J Roentgenol 201:332-339

26. Potretzke TA, Tan BR, Doyle MB, Brunt EM, Heiken JP, Fowler $\mathrm{KJ}$ (2016) Imaging features of biphenotypic primary liver carcinoma (hepatocho-langiocarcinoma) and the potential to mimic hepatocellular carcinoma: LI-RADS analysis of CT and MRI features in 61 cases. AJR Am J Roentgenol 207:25-31

27. Hwang J, Kim YK, Park MJ et al (2012) Differentiating combined hepatocellular and cholangiocarcinoma from mass-forming intrahepatic cholangiocarcinoma using gadoxetic acid-enhanced MRI. J Magn Reson Imaging 36:881-889

28. Wang Y, Yang Q, Li S et al (2019) Imaging features of combined hepatocellular and cholangiocarcinoma compared with those of hepatocellular carcinoma and intrahepatic cholangiocellular carcinoma in a Chinese population. Clin Radiol 74:407

29. De Campos RO, Semelka RC, Azevedo RM et al (2012) Combined hepatocellular carcinoma-cholangiocarcinoma: report of MR appearance in eleven patients. J Magn Reson Imaging 36:1139-1147

30. Jeon SK, Joo I, Lee DH et al (2019) Combined hepatocellular cholangiocarcinoma: LI-RADS v2017 categorisation for differential diagnosis and prognostication on gadoxetic acid-enhanced MR imaging. Eur Radiol 29:373-382

31. Lee HS, Kim MJ, An C (2019) How to utilize LR-M features of the LI-RADS to improve the diagnosis of combined hepatocellularcholangiocarcinoma on gadoxetate- enhanced MRI? Eur Radiol 29:2408-2416

32. Lin S, Ye F, Rong W et al (2019) Nomogram to assist in surgical plan for hepatocellular carcinoma: a prediction model for microvascular invasion. J Gastrointest Surg. https://doi.org/10.1007/s11605019-04140-0

33. Ryu T, Takami Y, Wada Y et al (2019) A clinical scoring system for predicting microvascular invasion in patients with hepatocellular carcinoma within the Milan criteria. J Gastrointest Surg 23:779 787

34. Tang Z, Liu WR, Zhou PY et al (2019) Prognostic value and predication model of microvascular invasion in patients with intrahepatic cholangiocarcinoma. J Cancer 10:5575-5584

35. Li R, Yang D, Tang CL et al (2016) Combined hepatocellular carcinoma and cholangio- carcinoma (biphenotypic) tumors: clinical characteristics, imaging features of contrast-enhanced ultrasound and computed tomography. BMC Cancer 16:158

36. Min JH, Kim YK, Lim S et al (2015) Prediction of microvascular invasion of hepatocellular carcinomas with gadoxetic acidenhanced MR imaging: impact of intra-tumoral fat detected on chemical-shift images. Eur J Radiol 84:1036-1043

37. Siripongsakun S, Lee JK, Raman SS et al (2012) MRI detection of intratumoral fat in hepatocellular carcinoma: potential biomarker for a more favorable prognosis. AJR Am J Roentgenol 199:10181025

38. Kutami R, Nakashima Y, Nakashima O, Shiota K, Kojiro M (2000) Pathomorphologic study on the mechanism of fatty change in small hepatocellular carcinoma of humans. J Hepatol 33:282-289

39. Lee S, Kang TW, Song KD et al (2019) Effect of microvascular invasion risk on early recurrence of hepatocellular carcinoma after surgery and radiofrequency ablation. Ann Surg. https://doi.org/10. 1097/SLA.0000000000003268

40. Choi JY, Lee JM, Sirlin CB (2014) CT and MR imaging diagnosis and staging of hepatocellular carcinoma: part II. Extracellular agents, hepatobiliary agents, and ancillary imaging features. Radiology 273:30-50

41. Sumie S, Nakashima O, Okuda K et al (2014) The significance of classifying micro-vascular invasion in patients with hepatocellular carcinoma. Ann Surg Oncol 21:1002-1009

Publisher's note Springer Nature remains neutral with regard to jurisdictional claims in published maps and institutional affiliations. 